\title{
Guarantee Industries Credit Evaluation Research of Risk Management Perspective
}

\author{
Yi Fang ${ }^{1}$, HuijunRen ${ }^{2}$, Longxing $\mathrm{Li}^{3}$ \\ Institute of Management Engineering, Zhengzhou University, \\ Zhengzhou, Henan Province, China, 450001
}

Keywords: Risk management; Credit evaluation; BP neural network

\begin{abstract}
In order to evaluate the credit status of the insured enterprise, from the perspective of guarantee company's risk management, according the evaluation indexes of the evaluation agencies both at home and abroad, this research constructs the credit guarantee evaluation index institutions used by themselves, and determines the weights of evaluation indexes by using FAHP. At last, this research designs the enterprise credit evaluation model based on the BP neural network and verifies the feasibility of the model through the demonstration analysis.
\end{abstract}

\section{Introduction}

Huge credit risks become the bottleneck of the development of financing guarantee agencies, seriously restrict the healthy development of the guarantee industries. There are a lot of sources of the risks, among them, the biggest risk comes from the insured enterprise, guarantee agencies obtain information mainly relies on providing by the companies themselves, there are serious information asymmetry. Besides, the credit rating technology of guarantee agencies is backward and the quality of the credit rating personnel is low, it is difficult for guarantee agencies to understand the actual risk status of the enterprise, so there is an enormous risk to provide security for them.

At present, the enterprise credit evaluation of our country is completed mainly by the commercial bank internal assessment and special credit evaluation institutions, but the rating contents, standards, the technologies of the different commercial banks are not identical and the classification difference is big. And most of the credit evaluation institutions of our country are not the independent legal person of real meaning, directly or indirectly attach themselves to government agencies or belong to the business unit, there are a lot of administrative intervention in the process of credit ratings, and lack the objective independence. ${ }^{[1]}$ Therefore, combining with the present situation of the guarantee companies own risk management needs, guarantee agencies establish a systematic enterprise credit evaluation system is more and more important.

\section{Constructing credit evaluation index system}

(1) The influence factors of the enterprise credit risk

There are a lot of influence factors of the enterprise credit risk, and in summary are divided into the quantitative factors and the qualitative factors. The qualitative factors mainly include enterprise credit performance ability, debt paying ability, profit ability, management and operation ability, market attraction, the product difference degree, management ability and environmental threat, innovation ability, enterprise quality, and the efficiency, etc. The quantitative factors mainly refer to the enterprise capital position, include the asset scale, liquidity, debt ratio and profit margins, etc.

(2) The choice of the evaluation indexes

Evaluation indexes, which are used by the different enterprise credit evaluation rating agencies are not identical, the United States moody's mainly include four aspects, the liquidity of enterprise assets, debt ratio, financial risk analysis and capital efficiency analysis about the enterprise credit evaluation. Standard \&poor's credit evaluation index system includes the three aspects of enterprise industry analysis, financial analysis and risk analysis. And the commercial banks of our country evaluate the customer credit rating level, mainly inspects the company's market competitiveness, liquidity of asset and asset management level, etc. ${ }^{[2]}$ 
According to the guarantee agencies own situation and the principle of index system design, and referencing the domestic and foreign research about evaluation index system design methods and ideas, from the view of risk management, this research divides the index system into three layers: one is the evaluation target, i.e., the company credit rating (target layer A); The second is evaluation elements (rule layer B),according to the company credit evaluation characteristics, this research selects the four evaluation elements of the market competition ability(B1), management level(B2), financial status(B3) and risk status(B4); The third layer is detailed and quantitative evaluation index (index layer $\mathrm{C}$ ), index layer is further segmentation evaluation factors, thus more specially displays evaluation elements and reflects evaluation target, this research includes twenty evaluation indicators, c11:Business environment (x1), c12: Market share (x2), c13: Company size (x3), c14: Development ability (x4), c15: Social approval (x5), c21:Management personnel quality (x6), c22:Management structure rationality (x7), c23: Assist ability (x8), c24: Work sense of responsibility (x9), c25: Management experiencex10), c31: Profit level(x11), c32: liquidity (x12), c33:Asset-liability ratio (x13), c34: Asset scale (x14), c35: Cash acquisition ability (x15), c41: Business risk (x16), c42: Debt paying ability (x17), c43: Financial risk (x18), c44: Credit risk(x 19), c45: Market risk (x20).

(3)Determining the index weight

There are a lot of methods of determining the index weight, combines with the characteristics of the enterprise credit evaluation, this research adopts the fuzzy analytic hierarchy process (FAHP) to determine the index weight, FAHP improves the weakness that consistency adjustment process is complicated of judgment matrix and improves the decision reliability based on the traditional analytic hierarchy process (AHP). ${ }^{[3]}$ According to the expert evaluation results, this research constructs the structure model of fuzzy consistent matrix, then single sorting, the results such as table 1.1 to table 1.5 shows.

Tab1.1. Target layer judgment matrix and sort results

\begin{tabular}{l|lllll}
\hline A & B1 & B2 & B3 & B4 & W \\
\hline B1 & 0.5 & 0.4 & 0.65 & 0.7 & 0.292 \\
B2 & 0.6 & 0.5 & 0.75 & 0.8 & 0.358 \\
B3 & 0.35 & 0.25 & 0.5 & 0.55 & 0.192 \\
B4 & 0.3 & 0.2 & 0.45 & 0.5 & 0.158 \\
\hline
\end{tabular}

Tab1.2. Rule layer B1 judgment matrix

\begin{tabular}{l|llllll}
\hline B1 & C11 & C12 & C13 & C14 & C15 & W \\
\hline C11 & 0.5 & 0.6 & 0.4 & 0.7 & 0.8 & 0.250 \\
C12 & 0.4 & 0.5 & 0.3 & 0.6 & 0.7 & 0.200 \\
C13 & 0.6 & 0.7 & 0.5 & 0.8 & 0.9 & 0.300 \\
C14 & 0.3 & 0.4 & 0.2 & 0.5 & 0.6 & 0.150 \\
C15 & 0.2 & 0.3 & 0.1 & 0.4 & 0.5 & 0.100 \\
\hline
\end{tabular}

Tab1.4. Rule layer B3 judgment matrix

\begin{tabular}{l|llllll}
\hline B3 & C31 & C32 & C33 & C34 & C35 & W \\
\hline C31 & 0.5 & 0.4 & 0.65 & 0.55 & 0.8 & 0.240 \\
C32 & 0.6 & 0.5 & 0.75 & 0.65 & 0.9 & 0.290 \\
C33 & 0.35 & 0.25 & 0.5 & 0.4 & 0.65 & 0.165 \\
C34 & 0.45 & 0.35 & 0.6 & 0.5 & 0.75 & 0.215 \\
C35 & 0.2 & 0.1 & 0.35 & 0.25 & 0.5 & 0.090 \\
\hline
\end{tabular}

Tab1.3. Rule layer B2 judgment matrix

\begin{tabular}{l|llllll}
\hline B2 & C21 & C22 & C23 & C24 & C25 & W \\
\hline C21 & 0.5 & 0.55 & 0.6 & 0.45 & 0.7 & 0.230 \\
C22 & 0.45 & 0.5 & 0.55 & 0.4 & 0.65 & 0.205 \\
C23 & 0.4 & 0.45 & 0.5 & 0.35 & 0.6 & 0.180 \\
C24 & 0.55 & 0.6 & 0.65 & 0.5 & 0.75 & 0.255 \\
C25 & 0.3 & 0.35 & 0.4 & 0.25 & 0.5 & 0.130 \\
\hline
\end{tabular}

Tab1.5. Rule layer B4 judgment matrix

\begin{tabular}{l|llllll}
\hline B4 & C41 & C42 & C43 & C44 & C45 & W \\
\hline C41 & 0.5 & 0.35 & 0.75 & 0.6 & 0.7 & 0.240 \\
C42 & 0.65 & 0.5 & 0.9 & 0.75 & 0.85 & 0.315 \\
C43 & 0.25 & 0.1 & 0.5 & 0.35 & 0.45 & 0.115 \\
C44 & 0.4 & 0.25 & 0.65 & 0.5 & 0.6 & 0.190 \\
C45 & 0.3 & 0.15 & 0.55 & 0.4 & 0.5 & 0.140 \\
\hline
\end{tabular}

According to the weight of the draw, we can get the total sort results of enterprise credit evaluation index weight, as is shown in table 1.6.

Tab1.6. The total sort results of enterprise credit evaluation index weight

\begin{tabular}{|l|c|c|c|c|c|c|c|c|c|c|}
\hline index & C11: & C12: & C13 & C34: & C35: & C41: & C42: & C43: & C24: & C25: \\
\hline weight & 0.073 & 0.058 & 0.088 & 0.044 & 0.029 & 0.082 & 0.073 & 0.064 & 0.091 & 0.047 \\
\hline index & C31: & C32: & C33: & C31: & C33: & C44: & C42: & C43: & C44: & C45: \\
\hline weight & 0.046 & 0.056 & 0.032 & 0.041 & 0.017 & 0.038 & 0.050 & 0.018 & 0.030 & 0.022 \\
\hline
\end{tabular}




\section{Constructing the risk assessment model}

(1) The methods of enterprise credit evaluation

There are a lot of research about enterprise credit assessment at home and abroad, and the methods of evaluation are so many, mainly have the factor analysis method, gray multi-level evaluation method and so on, because the starting point is different, the emphasis of evaluation is different, every method has its own advantages and disadvantages. ${ }^{[4]}$ But according to the characteristics of enterprise credit evaluation, this research adopts the method of combining the FAHP and BP neural network.

(2) The structure of the BP network model

Combining with the needs of the enterprise credit evaluation, this research adopts the three layers BP neural network which contains a hidden layer to establish enterprise credit evaluation model. First of all, the design of the input layer, this research uses the data of the twenty indexes after the standardization in level 3 as input layer neurons. Secondly, the design of the hidden layer, according to the training accuracy and evaluation needs, this research determines the number of hidden layer nodes to forty after many tests. Finally, the design of the output layer, in order to the needs of evaluate results and analysis, this research will set the BP network output layer neurons to 1 , and divides into five grades, A higher (0.800-1.000); B high (0.600-0.800); C medium (0.400-0.600); D low (0.200-0.400); E lower(0.000-0.200).

(3) The evaluation process of BP network model

Firstly, sample selection, according the evaluation index above in the table to collect sample, this research uses them as evaluation value of the insured enterprise. Secondly, making the processing of index standardization and dimensionless, the value of after treatment is between $\mathrm{o}$ and 1 . According the needs of the network training, this research is dimensionless to use the methods of the translation and poor transform, the process is as follows : ${ }^{[5]}$

When the data size and the target is accordant change, standardization formula is as shown in formular 1; else standardization formula is as shown in formular 2.

$$
\mathrm{f}_{i}=\frac{x_{i}-x_{\min }}{x_{\max }-x_{\min }}
$$

$$
\mathrm{f}_{i}=\frac{x_{\max }-x_{i}}{x_{\max }-x_{\min }}
$$

Finally, the network training and testing, the sample data is divided into two parts, one part is used as the training sample, and the network is trained until the precision meets the requirements; The other part of the data is used as a test sample, if the maximum relative error is within acceptable limits between output value and the expectations, the network has been training mature, next time, the evaluation results will be obtained only the input value of standardization can be inputted.

\section{Empirical Analysis}

Taking the 12 companies which cooperate with a guarantee company in Henan province for example, according the credit evaluation method in the front to obtain the every evaluation index score of every company, among them, the quantitative index value can be directly obtained, the qualitative index values will be obtained from the eguarantee company. Then making the input parameters standardization, calculating the comprehensive level results with the established target weight. The top ten groups are used as the training sample data, and the latter two groups are used as the test sample. As shown in table 3.1

Tab3.1. The normalized data of 12 companies' evaluation indexes

\begin{tabular}{|l|c|c|c|c|c|c|c|c|c|c|c|c|}
\hline & 1 & 2 & 3 & 4 & 5 & 6 & 7 & 8 & 9 & 10 & 11 & 12 \\
\hline $\mathrm{c} 11:$ & 0.535 & 0.232 & 0.566 & 0.354 & 0.515 & 0.374 & 0.758 & 0.273 & 0.859 & 0.343 & 0.444 & 0.646 \\
\hline $\mathrm{c} 12:$ & 0.545 & 0.343 & 0.556 & 0.848 & 0.465 & 0.455 & 0.394 & 0.444 & 0.848 & 0.444 & 0.556 & 0.778 \\
\hline $\mathrm{c} 13:$ & 0.616 & 0.505 & 0.788 & 0.636 & 0.687 & 0.566 & 0.717 & 0.576 & 0.828 & 0.768 & 0.636 & 0.879 \\
\hline $\mathrm{c} 14:$ & 0.434 & 0.333 & 0.636 & 0.566 & 0.566 & 0.354 & 0.424 & 0.455 & 0.778 & 0.545 & 0.475 & 0.758 \\
\hline $\mathrm{c} 15:$ & 0.535 & 0.313 & 0.545 & 0.505 & 0.485 & 0.253 & 0.343 & 0.343 & 0.778 & 0.455 & 0.556 & 0.556 \\
\hline
\end{tabular}




\begin{tabular}{|l|l|l|l|l|l|l|l|l|l|l|l|l|}
\hline c21: & 0.566 & 0.263 & 0.485 & 0.566 & 0.374 & 0.263 & 0.343 & 0.364 & 0.818 & 0.465 & 0.232 & 0.556 \\
\hline c22: & 0.646 & 0.333 & 0.667 & 0.636 & 0.495 & 0.384 & 0.384 & 0.263 & 0.818 & 0.576 & 0.545 & 0.677 \\
\hline c23: & 0.444 & 0.232 & 0.525 & 0.566 & 0.333 & 0.273 & 0.263 & 0.253 & 0.899 & 0.444 & 0.455 & 0.778 \\
\hline c24: & 0.535 & 0.475 & 0.778 & 0.818 & 0.646 & 0.646 & 0.636 & 0.232 & 0.778 & 0.778 & 0.535 & 0.848 \\
\hline c25: & 0.667 & 0.313 & 0.636 & 0.606 & 0.687 & 0.556 & 0.364 & 0.343 & 0.848 & 0.525 & 0.677 & 0.687 \\
\hline c31: & 0.465 & 0.273 & 0.505 & 0.545 & 0.556 & 0.475 & 0.263 & 0.202 & 0.788 & 0.465 & 0.747 & 0.586 \\
\hline c32: & 0.566 & 0.253 & 0.525 & 0.465 & 0.566 & 0.384 & 0.444 & 0.535 & 0.778 & 0.444 & 0.818 & 0.758 \\
\hline c33: & 0.667 & 0.333 & 0.455 & 0.737 & 0.677 & 0.495 & 0.475 & 0.343 & 0.818 & 0.556 & 0.455 & 0.677 \\
\hline c34: & 0.465 & 0.444 & 0.677 & 0.848 & 0.646 & 0.727 & 0.636 & 0.384 & 0.889 & 0.778 & 0.657 & 0.788 \\
\hline c35: & 0.263 & 0.263 & 0.364 & 0.535 & 0.444 & 0.394 & 0.384 & 0.273 & 0.788 & 0.354 & 0.313 & 0.687 \\
\hline c41: & 0.677 & 0.354 & 0.455 & 0.566 & 0.505 & 0.364 & 0.424 & 0.263 & 0.859 & 0.414 & 0.687 & 0.586 \\
\hline c42: & 0.778 & 0.283 & 0.384 & 0.636 & 0.444 & 0.384 & 0.384 & 0.232 & 0.818 & 0.343 & 0.364 & 0.889 \\
\hline c43: & 0.455 & 0.263 & 0.485 & 0.566 & 0.465 & 0.364 & 0.343 & 0.273 & 0.838 & 0.404 & 0.465 & 0.859 \\
\hline c44: & 0.384 & 0.364 & 0.778 & 0.768 & 0.687 & 0.556 & 0.556 & 0.374 & 0.869 & 0.657 & 0.788 & 0.788 \\
\hline c45: & 0.465 & 0.485 & 0.848 & 0.798 & 0.606 & 0.697 & 0.636 & 0.404 & 0.889 & 0.778 & 0.566 & 0.899 \\
\hline target & 0.554 & 0.338 & 0.598 & 0.625 & 0.540 & 0.445 & 0.475 & 0.346 & 0.827 & 0.539 & 0.542 & 0.734 \\
\hline
\end{tabular}

This research uses the GUI of MATLAB7.1 to evaluate the enterprise credit status. Target error precision is set to 0.001 and after 368 times of the network is training, the network error is close to 0.001 , at this time, we can think the network training already meet the requirements. Inputting the two data of test vector in the trained network evaluation model, we can obtain the credit level.

Checking sample of the actual output value and error, the output value of 11th company is 0.54273 and the target value is 0.542 , the evaluation results is $C$; the output value of 12 th company is 0.73391 and the target value is 0.734 , the evaluation results is B.It can be seen from the test results, they are basically the same between the above two companies' credit evaluation output results from the BP neural network and the results from the guarantee agencies' analysis and expected. It shows that the neural network model is suitable for the problem of enterprises' credit evaluation.

\section{Conclusion}

Through the study of existing sample mode, the enterprise credit evaluation system based on FAHP and BP neural network has won the guarantee agencies' knowledge and experience, later when the guarantee agencies need to evaluate the enterprise credit status, as long as input corresponding index data matrix to the trained BP neural network, BP network will reappear guarantee agencies' knowledge and experience and immediate response. Believing that along with the unceasing improvement of the guarantee agencies own risk management level and the science increasingly attention of enterprise credit assessment, the enterprise evaluation system based on FAHP and BP neural network will have greatly effect in guarantee agencies ' risk management.

\section{Acknowledgment}

Project name: The Small and Medium-sized Enterprises Credit Guarantee System Construction Research of Henan Province

Project number: 102400430030

\section{References}

[1] Yuanyuan Zhao.The perfection of legal system about our country's credit rating-in the perspective of financial security[J].Theoretical Exploration,2011 (3):142-144.

[2] Chungong Xiong,Research on the system of rating for Hi-tech enterprises[D]. Jiangsu university,Jiangsu,2005. 
[3] Zhi Gao,MuZi Li.Evaluation study of network-based growth competence for technology-oriented small and medium-sized enterprise based on Fussy-AHP[J]. Journal of dalian jiaotong university.2011 (1) :102-105.

[4] Bin Cai.Research on the enterprise's core competitiveness evaluation of High-tech companies based on improved BP Neural Network[D].Zhengzhou university, Zhengzhou, 2010.

[5] Yinglin Qin,Hongyan Li.A risk warning model of third-party logistics resource integration based on BP neural network[J]. Statistics and Decision, 2009(7): 31-33. 